\title{
O uso do equipamento de proteção individual em tempos de COVID-19: uma revisão
}

\section{da literatura}

\author{
The use of personal protection equipment in COVID-19 times: a literature review \\ El uso de equipo de protección personal en tiempos de COVID-19: una revisión de la literatura
}

Recebido: 08/02/2021 | Revisado: 16/02/2021 | Aceito: 20/02/2021 | Publicado: 28/02/2021

\author{
Fernanda da Silva Cardoso \\ ORCID: https://orcid.org/0000-0002-2533-765X \\ Universidade Federal do Estado do Rio de Janeiro, Brasil \\ E-mail: fernanda.silva2004@gmail.com \\ Denise de Assis Corrêa Sória \\ ORCID: https://orcid.org/0000-0003-0885-9226 \\ Universidade Federal do Estado do Rio de Janeiro, Brasil \\ E-mail: denisesoria@terracomb \\ Taís Veronica Cardoso Vernaglia \\ ORCID: https://orcid.org/0000-0003-3391-7301 \\ Universidade Federal do Estado do Rio de Janeiro, Brasil \\ E-mail: tais.vernaglia@unirio.br
}

\begin{abstract}
Resumo
O presente estudo propõe descrever e refletir sobre a utilização do equipamento de proteção individual (EPI) pelos(as) profissionais que atuam no transcorrer da cadeia assistencial de pessoas com suspeita ou confirmação de contaminação pelo novo Coronavírus. Trata-se de revisão integrativa, que analisou 10 (dez) artigos mapeados via Biblioteca Virtual em Saúde (BVS). Os resultados obtidos evidenciam ser oportuna a abordagem, segundo a literatura em questão, sobre o tema no atual contexto da pandemia de COVID-19, pois os estudos mapeados ressaltam, para os indivíduos que vivenciam os cuidados acerca da doença, a urgente compreensão sobre o uso do EPI, ao passo que auxilia a área da Enfermagem e demais profissionais da saúde no manejo adequado dos equipamentos. Por fim, as conclusões destacam o sistemático e agravado quadro de adoecimento, morte e precarização da atuação deste grupo de profissionais, sobretudo atualmente, durante a pandemia COVID-19.

Palavras-chave: Equipamento de proteção individual; Enfermagem; COVID-19; Revisão integrativa.
\end{abstract}

\begin{abstract}
This study proposes to describe and reflect on the use of personal protective equipment (PPE) by professionals working in the care chain of people with suspected or confirmed contamination by the new Coronavirus. This is integrative review, which analyzed a set of 10 (ten) articles mapped through the Virtual Health Library (VHL). With the conclusion of the study, the results obtained show that it is opportune to approach, according to the literature in question, the topic in the current context of the pandemic of COVID-19, as the mapped studies emphasize, for individuals who experience care about the disease, the urgent understanding of the importance of using PPE, while helping the Nursing area and other health professionals in the proper handling of equipment. Finally, the conclusions highlight the systematic and aggravated picture of illness, death and the precarious performance of this group of professionals, especially today, during the pandemic of COVID-19.
\end{abstract}

Keywords: Personal protective equipment; Nursing; COVID-19; Integrative review.

\section{Resumen}

Este estudio propone describir y reflexionar sobre el uso de equipos de protección personal (EPI) por parte de los profesionales que laboran en la cadena asistencial de personas con sospecha o confirmación de contaminación por el nuevo Coronavirus. Se trata de revisión integrador, que analizó un conjunto de 10 (diez) artículos mapeados a través de la Biblioteca Virtual en Salud (BVS). Con la conclusión del estudio, los resultados obtenidos muestran que es oportuno abordar, de acuerdo con la literatura en cuestión, el tema en el contexto actual de la pandemia de COVID-19, como enfatizan los estudios mapeados, para individuos que experimentan cuidados por la enfermedad. , la urgente comprensión de la importancia de utilizar EPI, al tiempo que se ayuda al área de Enfermería y otros profesionales de la salud en el correcto manejo de los equipos. Finalmente, las conclusiones destacan el cuadro sistemático y agravado de enfermedad, muerte y el precario desempeño de este grupo de profesionales, especialmente hoy, durante la pandemia de COVID-19.

Palabras clave: Equipo de protección personal; Enfermería; COVID-19; Revisión integradora. 


\section{Introdução}

O presente estudo propõe descrever e refletir sobre a utilização do equipamento de proteção individual (EPI) pelos(as) profissionais que atuam no transcorrer da cadeia assistencial de pessoas com suspeita ou confirmação de contaminação pelo novo Coronavírus.

Durante a pandemia Covid-19, mostrou-se indispensável manter em atividade o maior número de profissionais, visando a minimização de impactos negativos desta situação de pandemia na sociedade, os cuidados com a prevenção de doenças e promoção da saúde entre os(as) trabalhadores(as) devem ser assumidos enquanto prioridade. Assegurar o acesso qualitativo aos Equipamentos de Proteção Individual (EPI) em quantidade suficiente e com eficácia, assim como a capacitação dos trabalhadores dos fluxos de atendimento e para o uso correto das barreiras à exposição, é essencial (Gallasch et al., 2020).

$\mathrm{Na}$ prestação de serviços pelos(as) profissionais, o conceito de biossegurança necessita estar presente e deve ser priorizado, devendo os serviços de saúde atenderem às Normas Regulamentadoras - NR, sendo a NR 32, do Ministério do Trabalho e Emprego, que estabelece as diretrizes básicas para a implementação de medidas de proteção à segurança e a saúde dos(as) trabalhadores(as) em serviços de saúde. Deste modo, a Norma recomenda, para cada situação de risco, a adoção de medidas preventivas e a capacitação dos(as) trabalhadores(as) para o trabalho seguro (Brasil, 2005).

No Brasil, os EPI devem estar regularizados junto aos órgãos certificadores e à Agência Nacional de Vigilância Sanitária (ANVISA). Tal órgão, motivado pela atual situação de emergência de saúde pública internacional relacionada à COVID-19, por meio da Resolução da Diretoria Colegiada (RDC) no 356/2000, simplificou os requisitos para fabricação, importação e aquisição de dispositivos médicos prioritários e de máscaras cirúrgicas, respiradores particulados N95, PFF2 ou equivalentes, utilizados em serviços de saúde (Brasil, 2020).

De tal modo, o uso de EPI deve considerar o nível de cuidado e tipo de atividade a ser executada, a saber: triagem, amostra para diagnóstico laboratorial, caso suspeito ou confirmado de 2019-nCov que requer internação na unidade de saúde e sem procedimento gerador de aerossol (PGA), e, por fim, caso suspeito ou confirmado de 2019-nCov que requer internação na unidade de saúde e PGA (Brasil, 2020; Who, 2020). Recomenda-se adotar precauções-padrão, de contato e de transmissão por gotículas a todos os casos suspeitos ou confirmados de Covid-19 e, em situações específicas, utilizar precauções por aerossol (Who, 2020b).

Portanto, os(as) profissionais da saúde envolvidos(as) nos cuidados diretos de pacientes devem usar: batas cirúrgicas, luvas, máscara cirúrgica e proteção dos olhos (óculos de proteção ou máscara facial) (Anvisa, 2020c; Who, 2020c). No mesmo sentido, não devem ser utilizadas duas luvas (sobrepostas) e o óculos de proteção/protetor facial devem ser exclusivo de cada profissional e passar por limpeza e desinfecção imediatamente após o uso (Anvisa, 2020c).

Igualmente, se as batas cirúrgicas não forem resistentes a fluidos, deve-se utilizar, também, aventais e somente nos casos de procedimento gerador de aerossol (intubação traqueal, ventilação não invasiva, traqueostomia, ressuscitação cardiopulmonar, entre outros), é que se deve usar respiradores (máscaras N95, PFF2 ou padrão equivalente) junto aos demais EPI's (Janssen, Zhuang, Shaffer, 2014).

Complementarmente, as máscaras cirúrgicas devem ter alta resistência ao fluido; boa respirabilidade; no mínimo, uma camada interna e externa; e, obrigatoriamente, um elemento filtrante. Paralelamente, devem ser confeccionadas de forma a cobrir adequadamente a área do nariz e da boca do usuário, possuindo clipes nasais constituídos de material maleável, de modo a permitir ajustes adequados ao contorno do nariz e das bochechas do(a) usuário(a) (Brasil, 2020).

Tais máscaras não devem ser sobreposta à máscara N95 ou equivalente, pois além de não garantirem proteção de filtração ou de contaminação, contribui com o desperdício de mais um EPI (Anvisa, 2020). No caso das máscaras N95/PFF2 ou equivalente, é recomendado que se proceda a troca, quando estas estiverem saturadas, sujas ou úmidas, amassadas ou com vincos (Brasil, 2020). 
Para reutilizar a máscara N95/PFF2 ou equivalente, é preciso protegê-la da exposição às gotículas expelidas pelo paciente, isso pode ser feito utilizando-se o protetor facial; deve-se sempre inspecionar visualmente a máscara antes de cada uso, para avaliar sua integridade e realizar o teste de vedação. Caso não seja possível realizar uma verificação bem-sucedida da vedação da máscara à face do(a) trabalhador(a), a máscara deverá ser descartada imediatamente. Ressalta-se que a reutilização é uma prática limitada, pois existem restrições que balizam o número de vezes de reuso (Ferioli et al., 2020).

Cabe às instituições de saúde, do nível primário à alta complexidade, o treinamento dos(as) profissionais (especialmente, sobre técnicas de paramentação e desparamentação), a supervisão sobre uso dos EPI, a manutenção e a reposição dos EPI conforme indicação do fabricante (Brasil, 2020). Assim, é imprescindível garantir a proteção dos(as) profissionais de saúde contra o vírus, a fim de garantir que eles(as) não atuem como vetores de transmissão, além de evitar seu adoecimento, que pode preservar a manutenção do atendimento à população necessitada (Chang et al., 2020).

Diante do quadro apresentado, destaca-se a importância de os(as) profissionais de saúde, sobretudo enfermeiros(as) e demais profissionais da enfermagem que prestam cuidados, continuamente na linha de frente, reconhecerem a importância do uso de equipamentos de proteção individual (EPI) adequados para minimizar a disseminação do vírus, adoecimento e afastamento das suas atividades laborais.

Objetivo geral: compreender a utilização do equipamento de proteção individual pelos profissionais que atuam no transcorrer da cadeia assistencial de pessoas com suspeita ou confirmação de contaminação pelo novo COVID-19. São objetivos específicos: mapear estudos recentes que contemplem a utilização do equipamento de proteção individual (EPI) e sua importância para profissionais que atuam no transcorrer da cadeia assistencial; identificar o uso adequado do equipamento de proteção individual na proteção e controle da COVID-19; e, discutir a utilização de equipamento de proteção individual e sua importância para profissionais que atuam frente a pacientes com suspeita ou confirmação de contaminação pelo COVID-19.

\section{Metodologia}

Trata-se de estudo de revisão integrativa da literatura, para reunir e sintetizar achados de estudos realizados, mediante diferentes métodos, com intuito de contribuir para o aprofundamento do conhecimento relativo ao tema investigado (Soares et al., 2014). Em aspectos gerais: A revisão integrativa é tipo de estudo, que proporciona síntese de conhecimento e incorporação da aplicabilidade de resultados de estudos significativos à prática (Souza, Silva, Carvalho, 2010, p. 102).

O presente estudo foi fundamentado em seis etapas, conforme Souza, Silva e Carvalho (2010): 1) Elaboração da pergunta norteadora; 2) Busca na literatura; 3) Coleta de dados; 4) Análise crítica dos estudos incluídos; 5) Discussão dos resultados; 6) Apresentação da revisão integrativa.

Assim, a revisão realizada partiu da seguinte questão de pesquisa: Quais as evidências científicas relacionadas à utilização do equipamento de proteção individual (EPI) pelos (as) profissionais de enfermagem frente às pessoas com suspeita ou confirmação de contaminação pelo COVID-19?

Para se iniciar essa pesquisa, foram realizadas buscas na base de dados da Biblioteca Virtual em Saúde (BVS), para encontrar publicações que embasassem a temática escolhida. A Biblioteca Virtual em Saúde visa convergir as redes temáticas no campo da investigação em saúde e integrar importante fonte de informação neste campo do saber. Assim, é importante repositório e tem fortalecido e dado considerável visibilidade aos estudos desenvolvidos, por meio de acesso ao seu conteúdo. As fontes de informação da BVS obedecem ao controle de qualidade científica e, assim, são recorrentemente citadas por pesquisadores(as) que investigam os mais variados temas ligados à saúde. Portanto, este repositório foi eleito por oferecer importante conjunto de informações cientificas e técnica interligadas às Ciências da Saúde, sejam elas publicadas no Brasil ou sobre o contexto brasileiro.

Foram utilizados os seguintes descritores: ((Equipamento de Proteção Individual)) OR ((Equipamento de Proteção 
Pessoal)) OR ((Equipamentos de Proteção Individual)) OR ((Equipamentos de Proteção Pessoal)) AND ((Infecções por Coronavírus)) OR ((COVID-19)). A combinação descrita resultou em 70 trabalhos. Associado a isso, utilizamos filtros secundários relacionados ao "Assunto principal”: ‘Equipamento de Proteção Individual', que reuniu 53 trabalhos, e 'Infecções por Coronavírus', com 43 estudos publicados. Com a utilização do conjunto de descritores mencionado anteriormente foram localizados 21 artigos científicos na MEDLINE e 25 na LILACS, 10 textos na BDENF - Enfermagem, 13 no Coleciona SUS, 03 no BBO - Odontologia, 10 no SCIELO e no WHO IRIS, 02 no PAHO-IRIS e CVSP - Brasil e 01 Sec. Munic. Saúde SP, num total de 70 artigos que abordam de maneira geral a temática em torno do uso de equipamento de proteção individual (EPI) no universo de atuação frente a pessoas com suspeita ou confirmação de contaminação pelo COVID-19.

Após, a aplicação dos critérios de inclusão: publicações em português e inglês, máximo cinco anos de publicação (20152020) e possuir objeto de estudo condizente com a temática proposta, restaram 10 (dez) artigos científicos. Assim, consideramos textos apresentados integralmente, nos idiomas mencionados anteriormente, e cujo título e/ou resumo fizessem referência à temática.

Os critérios de exclusão aplicados foram: repetições nas bases de dados, artigos de opinião, editoriais, estudos de casos, cartas ao editor e notas prévias.

A análise foi construída via organização, síntese das principais informações, leitura detalhada dos estudos e, por fim, agrupamento em temáticas, nas quais foram destacadas como as principais contribuições do estudo e levantamento realizado em torno dos temas em questão. Por não se tratar de uma pesquisa realizada com seres humanos, não se fez necessária a submissão e aprovação desta pelo Comitê de Ética e Pesquisa (CEP). Neste estudo são respeitados os conceitos, as definições e as ideias dos(as) autores(as) das publicações, de forma a não haver nenhuma alteração do conteúdo original em prol de benefícios à revisão, mantendo-se os direitos autorais dos mesmos.

\section{Resultados}

Para melhor compreensão e análise sistemática dos artigos, realizamos leitura individualizada e minuciosa para melhor análise do conteúdo, com finalidade de identificar a ordem das informações e realizar levantamento das possíveis conexões entre eles.

A partir dos artigos analisados e conforme a proposta do objetivo do estudo, foram selecionados como categoria de estudo de análise: Escassez e qualidade dos equipamentos de proteção individual (EPI) para profissionais de saúde durante a pandemia e treinamento para utilização do equipamento de proteção individual (EPI) e condições de trabalho dos profissionais de saúde durante a pandemia, conforme apresentado a seguir.

Em linhas gerais, as categorias de análise eleitas correspondem aos grandes temas recorrentes nas pesquisas mapeadas e serão utilizadas como forma de agrupar as referidas pesquisas e no encaminhamento do processo de descrição e análise dos achados. Abaixo apresentamos as categorias eleitas e, em seguida, o perfil dos estudos que correspondem a cada uma delas. 
Quadro 1 - Sumarização dos artigos que constituem a amostra da revisão de literatura.

\begin{tabular}{|l|l|}
\hline \multicolumn{1}{|c|}{ Categoria de Análise } & \multicolumn{1}{c|}{ Perfil dos Estudos Mapeados } \\
\hline $\begin{array}{l}\text { Escassez e qualidade dos equipamentos de proteção } \\
\text { individual (EPI) para profissionais de saúde durante a } \\
\text { pandemia e treinamento para utilização do equipamento } \\
\text { de proteção individual (EPI). }\end{array}$ & $\begin{array}{l}\text { Os textos relacionados com a presente categoria de análise investigam } \\
\text { diferentes processos, rotinas e práticas, no âmbito da saúde, voltadas, } \\
\text { sobretudo, à conscientização, orientação e implementação de ações voltadas ao } \\
\text { uso de equipamento de proteção individual (EPI). }\end{array}$ \\
\hline $\begin{array}{l}\text { Condições de trabalho dos profissionais de saúde durante } \\
\text { a pandemia. }\end{array}$ & $\begin{array}{l}\text { Na presente categoria os textos catalogados estão relacionados com a análise e } \\
\text { a problematização do adoecimento, morte e precariedade da prestação de } \\
\text { serviços por profissionais de saúde durante a pandemia de COVID-19 e a } \\
\text { relação com o uso de equipamento de proteção individual (EPI). }\end{array}$ \\
\hline
\end{tabular}

Fonte: Autoras.

Após o processo de agrupamento dos artigos com base nas categorias de análise anteriormente mencionadas, é indicado, abaixo, o quadro de sumarização dos textos que compõe o presente estudo, sendo destacadas as suas principais características:

Quadro 2 - Sumarização dos artigos que constituem a amostra da revisão de literatura.

\begin{tabular}{|c|c|c|c|}
\hline Título do texto & Objetivo do estudo & $\begin{array}{l}\text { Conteúdo } \\
\text { temático }\end{array}$ & Resultado do estudo \\
\hline $\begin{array}{l}\text { Equipamento de Proteção } \\
\text { Individual na pandemia por } \\
\text { coronavírus: treinamento com } \\
\text { Prática Deliberada em Ciclos } \\
\text { Rápidos }\end{array}$ & $\begin{array}{l}\text { Discutir a aplicação da Prática } \\
\text { Deliberada em Ciclos Rápidos } \\
\text { para o treinamento de } \\
\text { paramentação } \\
\text { desparamentação no contexto da } \\
\text { COVID-19 e estruturar um guia } \\
\text { prático para a aplicação nesta } \\
\text { conjuntura }\end{array}$ & $\begin{array}{l}\text { Treinamento } \\
\text { para utilização } \\
\text { adequada do } \\
\text { equipamento de } \\
\text { proteção } \\
\text { individual. }\end{array}$ & $\begin{array}{l}\text { Maximização do tempo em Prática } \\
\text { Deliberada, feedback com evidência e } \\
\text { segurança psicológica são os princípios } \\
\text { desta estratégia. A dinâmica envolve } \\
\text { repetições e feedbacks. O guia de aplicação } \\
\text { apresenta a sequência de ações para } \\
\text { paramentação e desparamentação. }\end{array}$ \\
\hline $\begin{array}{l}\text { Recomendações para a utilização de } \\
\text { máscaras em ambiente hospitalar } \\
\text { durante a pandemia ocasionada pelo } \\
\text { Coronavírus }\end{array}$ & $\begin{array}{l}\text { Analisar na literatura as } \\
\text { diferentes recomendações } \\
\text { nacionais e internacionais sobre } \\
\text { o uso de máscaras no ambiente } \\
\text { hospitalar durante a pandemia } \\
\text { ocasionada por Coronavírus. }\end{array}$ & $\begin{array}{l}\text { Utilização } \\
\text { adequada do } \\
\text { equipamento, } \\
\text { conforme a } \\
\text { assistência } \\
\text { prestada. }\end{array}$ & $\begin{array}{l}\text { Diferentes tipos de máscaras vêm sendo } \\
\text { empregadas como meio de proteção durante } \\
\text { o enfrentamento da pandemia. Para sua } \\
\text { utilização, os profissionais de saúde devem } \\
\text { seguir boas práticas de uso, combinada as } \\
\text { outras medidas de proteção. }\end{array}$ \\
\hline $\begin{array}{l}\text { Utilização de máscaras: indicações } \\
\text { de uso e manejo durante a pandemia } \\
\text { da COVID-19 }\end{array}$ & $\begin{array}{l}\text { Discutir a forma correta de } \\
\text { utilização, indicações de uso, } \\
\text { eficácia e manejo de cada tipo de } \\
\text { máscara, assim como sugerir, } \\
\text { conforme evidências científicas } \\
\text { e recomendações de órgãos de } \\
\text { saúde, alternativas eficazes para } \\
\text { aumentar a vida útil deste } \\
\text { produto. }\end{array}$ & $\begin{array}{ll}\text { Escassez } & \text { do } \\
\text { equipamento } & \text { de } \\
\text { proteção } & \\
\text { individual } & \end{array}$ & $\begin{array}{l}\text { Máscaras de proteção respiratória podem ser } \\
\text { reutilizadas se estiverem íntegras, limpas e } \\
\text { secas. Para aumentar a durabilidade de } \\
\text { máscaras cirúrgicas, sugere-se a utilização } \\
\text { com papel toalha para absorver a umidade. } \\
\text { Máscaras de tecido são recomendadas, desde } \\
\text { que sejam lavadas adequadamente para } \\
\text { reutilização. }\end{array}$ \\
\hline
\end{tabular}




\begin{tabular}{|c|c|c|c|}
\hline $\begin{array}{l}\text { Pandemia de COVID-19 e o uso } \\
\text { racional de equipamentos de } \\
\text { proteção individual }\end{array}$ & $\begin{array}{l}\text { Descrever as recomendações } \\
\text { sobre o uso racional e seguro dos } \\
\text { equipamentos de proteção } \\
\text { individual (EPI) no transcorrer } \\
\text { da cadeia assistencial de pessoas } \\
\text { com suspeita ou confirmação de } \\
\text { contaminação pelo novo } \\
\text { coronavírus. }\end{array}$ & $\begin{array}{lr}\text { Escassez } & \mathrm{e} \\
\text { qualidade } & \text { do } \\
\text { equipamento } & \text { de } \\
\text { proteção } & \\
\text { individual } & \end{array}$ & $\begin{array}{l}\text { Neste contexto, diversos países, inclusive o } \\
\text { Brasil, têm registrado falhas na proteção dos } \\
\text { trabalhadores da saúde por conta da escassez } \\
\text { destes equipamentos ou devido à equívocos } \\
\text { na paramentação ou desparamentação. }\end{array}$ \\
\hline $\begin{array}{l}\text { Personal protective equipment for } \\
\text { preventing highly infectious } \\
\text { diseases due to exposure to } \\
\text { contaminated body fluids in } \\
\text { healthcare staff }\end{array}$ & $\begin{array}{l}\text { Avaliar qual tipo de EPI de } \\
\text { corpo inteiro e qual método de } \\
\text { colocação ou retirada do EPI tem } \\
\text { o menor risco de contaminação } \\
\text { ou infecção para o profissional } \\
\text { de saúde e quais métodos de } \\
\text { treinamento aumentam a } \\
\text { conformidade com os protocolos } \\
\text { de EPI. }\end{array}$ & $\begin{array}{ll}\text { Escassez } & \text { do } \\
\text { equipamento } & \text { de } \\
\text { proteção } & \\
\text { individual } & \end{array}$ & $\begin{array}{l}\text { Cobrir mais o corpo leva a uma melhor } \\
\text { proteção. No entanto, como isso geralmente } \\
\text { está associado a maior dificuldade em } \\
\text { colocar e remover o EPI, e o EPI é menos } \\
\text { confortável, pode levar a mais } \\
\text { contaminação. Tipos de PPE mais } \\
\text { respiráveis podem levar a níveis semelhantes } \\
\text { de contaminação, mas são mais } \\
\text { confortáveis. A contaminação foi comum } \\
\text { em metade dos estudos, apesar da melhora } \\
\text { do EPI. }\end{array}$ \\
\hline $\begin{array}{l}\text { Barriers and facilitators } \\
\text { to healthcare workers' adherence } \\
\text { with infection prevention and } \\
\text { control (IPC) guidelines for } \\
\text { respiratory infectious diseases: a } \\
\text { rapid qualitative evidence synthesis }\end{array}$ & $\begin{array}{l}\text { Identificar barreiras e } \\
\text { facilitadores para a adesão } \\
\text { dos profissionais de saúde } \\
\text { às diretrizes da prevenção e } \\
\text { controle de infecção (IPC) } \\
\text { para doenças infecciosas } \\
\text { respiratórias. }\end{array}$ & 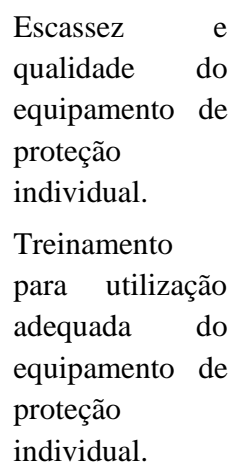 & $\begin{array}{l}\text { A comunicação clara sobre as diretrizes do } \\
\text { IPC foi considerada vital. Mas os } \\
\text { profissionais de saúde apontaram a falta de } \\
\text { treinamento sobre a infecção em si e sobre } \\
\text { como usar o EPI. Eles também achavam que } \\
\text { era um problema quando o treinamento não } \\
\text { era obrigatório. }\end{array}$ \\
\hline $\begin{array}{l}\text { Personal protective equipment } \\
\text { during the coronavirus disease } \\
\text { (COVID) } 2019 \text { pandemic - a } \\
\text { narrative review }\end{array}$ & $\begin{array}{l}\text { Esta revisão visa acrescentar } \\
\text { alguma clareza a respeito dos } \\
\text { modos de transmissão do } \\
\text { COVID - 19, qual EPI é } \\
\text { recomendado, quando e por quê. }\end{array}$ & $\begin{array}{ll}\text { Escassez } & \text { do } \\
\text { equipamento } & \text { de } \\
\text { proteção } & \\
\text { individual } & \end{array}$ & $\begin{array}{l}\text { Esta revisão relata que os vestidos oferecem } \\
\text { melhor proteção do que os aventais; que a } \\
\text { troca apoiada por instruções verbais reduz os } \\
\text { erros; e um único estudo de simulação } \\
\text { sugere que o uso de um respirador } \\
\text { purificador de ar pressurizado pode reduzir a } \\
\text { contaminação em comparação com o PPE } \\
\text { mais convencional. }\end{array}$ \\
\hline $\begin{array}{l}\text { The Italian coronavirus disease } \\
2019 \text { outbreak: recommendations } \\
\text { from clinical practice }\end{array}$ & $\begin{array}{l}\text { Descrevemos os principais } \\
\text { elementos do manejo clínico, } \\
\text { incluindo: oxigenoterapia } \\
\text { segura; gerenciamento das vias } \\
\text { aéreas; equipamento de proteção } \\
\text { pessoal; e aspectos não técnicos } \\
\text { do atendimento de pacientes } \\
\text { com diagnóstico de doença } \\
\text { coronavírus } 2019 \text {. }\end{array}$ & $\begin{array}{l}\text { Escassez do } \\
\text { equipamento de } \\
\text { proteção } \\
\text { individual. } \\
\text { Treinamento } \\
\text { para utilização } \\
\text { adequada do } \\
\text { equipamento de } \\
\text { proteção } \\
\text { individual. } \\
\text { Condições de } \\
\text { trabalho frente à } \\
\text { pandemia. }\end{array}$ & $\begin{array}{l}\text { Embora a Organização Mundial da Saúde } \\
\text { (OMS) emita atualizações regulares sobre a } \\
\text { transmissão de doenças e o uso adequado de } \\
\text { EPI, a realidade, conforme observado no } \\
\text { mesmo relatório da OMS, é que tal surto em } \\
\text { grande escala resultou em uma escassez de } \\
\text { EPI para profissionais de saúde. }\end{array}$ \\
\hline
\end{tabular}




\begin{tabular}{|c|c|c|c|}
\hline $\begin{array}{l}\text { De cuidador a paciente: na } \\
\text { pandemia da COVID-19, quem } \\
\text { defende e cuida da enfermagem } \\
\text { brasileira? }\end{array}$ & $\begin{array}{l}\text { Refletir sobre a saúde do } \\
\text { trabalhador de enfermagem } \\
\text { diante da crise da pandemia } \\
\text { pela COVID-19. }\end{array}$ & $\begin{array}{l}\text { Escassez do } \\
\text { equipamento de } \\
\text { proteção } \\
\text { individual. } \\
\text { Condições de } \\
\text { trabalho frente à } \\
\text { pandemia. }\end{array}$ & $\begin{array}{l}\text { Portanto, o trabalhador de enfermagem se vê } \\
\text { diante de duas situações paradoxais, uma } \\
\text { pende para a sua responsabilidade como } \\
\text { profissional e a sua importância no sistema } \\
\text { de saúde, seja no serviço privado ou no } \\
\text { público, e a outra como ser humano } \\
\text { intimidado em um campo permeado por } \\
\text { fragilidades, dúvidas, riscos, mudanças } \\
\text { assistenciais, escassez de insumos, onde } \\
\text { vulnerabilidades em relação a sua saúde e de } \\
\text { seus familiares são afloradas. }\end{array}$ \\
\hline $\begin{array}{l}\text { Condições de trabalho e o impacto } \\
\text { na saúde dos profissionais de } \\
\text { enfermagem frente a COVID-19 }\end{array}$ & $\begin{array}{l}\text { Refletir sobre as condições de } \\
\text { trabalho dos profissionais de } \\
\text { enfermagem no enfrentamento } \\
\text { ao novo coronavírus e apontar o } \\
\text { impacto na vida desses } \\
\text { profissionais em meio à } \\
\text { pandemia. }\end{array}$ & $\begin{array}{l}\text { Escassez do } \\
\text { equipamento de } \\
\text { proteção } \\
\text { individual. } \\
\text { Condições de } \\
\text { trabalho frente à } \\
\text { pandemia. }\end{array}$ & $\begin{array}{l}\text { As fragilidades encontradas no cotidiano } \\
\text { laboral dos profissionais de enfermagem } \\
\text { são descritas pela literatura nacional e } \\
\text { internacional, nas quais estão incluídas as } \\
\text { más condições de trabalho, sobrecarga física } \\
\text { e mental, baixa remuneração e ausência de } \\
\text { Equipamentos de Proteção Individual } \\
\text { adequados para o enfrentamento desse } \\
\text { agravo. }\end{array}$ \\
\hline
\end{tabular}

Fonte: Autoras.

\section{Discussão}

A seguir apresentamos os resultados e discussões construídos a partir da análise descritiva e dialógica dos estudos mapeados e categorizados a partir dos descritos mencionados anteriormente.

\subsection{Escassez e qualidade dos equipamentos de proteção individual (EPI) para profissionais de saúde durante a pandemia}

O aumento exponencial de casos de infecção ocasionada pelo coronavírus (COVID-19) tem requerido dos diversos países afetados esforços para garantir a provisão às equipes de saúde de insumos necessários para o enfrentamento da pandemia. Tais ações têm levado em consideração, sobretudo, as estimativas epidemiológicas da quantidade de pacientes com COVID-19 que necessitarão de assistência médica (Chan, et al.; 2020).

A China é, atualmente, o principal produtor e exportador destes tipos de materiais, mas sendo o primeiro epicentro do SARS-CoV2, o país teve a produção e venda destes equipamentos afetadas. Ao serem retomadas as atividades, as indústrias chinesas conviveram com o elevado número de solicitações advindas de inúmeras partes do mundo, necessitando recuperar a plena capacidade de fabricação e, sobretudo, aumentá-la. Diante da dificuldade de acesso, dos elevados preços e da qualidade duvidosa de alguns EPI, foi preciso avaliar o planejamento de compra, fabricantes, intermediários de terceiros (quando aplicável) e os termos contratuais para a organização das compras. Nesse sentido, foi preciso considerar planos de logística ampliados para garantir a entrega dos materiais em tempo hábil, pois sua recepção sempre foi premente (CDCP, 2020).

A velocidade e o avanço descontrolado da COVID-19 em todo o mundo colocaram em xeque o gerenciamento da cadeia de abastecimento de equipamentos de proteção individual, o planejamento, a implementação, o controle do fluxo e, particularmente, o armazenamento eficiente dos equipamentos para suprir as demandas nas diferentes esferas da cadeia assistencial. Foi evidente, em diversos momentos, a falta de planejamento logístico para a definição de ações e a adoção de intervenções estratégicas de preservação para com a saúde dos(as) trabalhadores(as) que prestam cuidados a pacientes com suspeita ou confirmação da infecção pelo COVID-19, frente à escassez dos equipamentos de proteção individual (EPI).

Um importante fator de risco eminente para manutenção e diminuição na disseminação do contágio entre aos(as) 
trabalhadores(as) da linha de frente aponta para a qualidade dos equipamentos de proteção individual, observado a partir dos estudos analisados, mostra as contestáveis, causando falhas e negligenciado a proteção desses trabalhadores/as, o que tem impactado diretamente na contaminação, absenteísmo e morte desses(as) profissionais.

Nesse sentido, segundo a Associação Brasileira de Hospitais Privados, seus hospitais possuem apenas $20 \%$ do estoque necessário de EPI. Dados apurados pela Associação Brasileira de Medicina, por meio do registro de denúncias dos(as) profissionais, revelam, igualmente, o dimensionamento da falta de EPI: luva (28\%), máscara (87\%), gorro (46\%), óculos ou face shield (72\%), capote impermeável (66\%), outros (19\%) (ABM, 2020).

A escassez de EPI é um problema que afetou o sistema de saúde em todo o mundo, conforme relevam os estudos analisados, levando os gestores de diversos países a disputarem a compra de equipamentos de proteção individual para suprir a grande demanda que a pandemia ocasionou nos diferentes níveis de atenção à saúde. Nesse sentido, era previsível que, em momentos de pandemia, a provisão de equipamentos de proteção individual (EPI) atravessaria períodos de escassez e desabastecimento em setores que demandam a sua utilização em maior quantidade. No entanto, a situação tornou-se mais preocupante quando associado aos danos que o déficit pôde ocasionar aos(às) profissionais que atuam na linha de frente contra o COVID-19.

Esta é uma questão principal vista a partir dos estudos mapeados, uma vez que dificuldades no acesso de EPIs adequados contribuem para o aumento da exposição entre os(as) trabalhadores(as) e que acarretam a disseminação do vírus, no afastamento desses(as) profissionais e o risco eminente para familiares e comunidade. Prover condições seguras para os(as) profissionais que atuam no combate ao COVID-19, com EPIs de qualidade e quantidade adequada, é o ponto de partida problematizado nos estudos analisados para a garantia básica de segurança na prestação da assistência desses(as) profissionais.

Em contexto de escassez têm sido questionadas, nas pesquisas, estratégias de uso estendido e de reuso limitado de equipamentos. Isso é feito alertando para o aumento de riscos de contaminação e abrindo polêmicas. Tomadores de decisão precisam, assim, explicitar critérios usados nessas situações (Berlinger, et al.; 2020).

Em caráter excepcional, isso é, em situações de carência de insumos, para atender a demanda da epidemia de Covid-19, que estes EPI's poderão ser usados por período maior ou por um número de vezes ampliado que o previsto pelo fabricante, desde que pelo(a) mesmo(a) profissional e cumprindo as rotinas estabelecidas pelas Comissões de Controle de Infecção Hospitalar do Serviço de Saúde. Os serviços de saúde devem, então, definir um protocolo para orientar os(as) profissionais sobre o uso prolongado dos respiradores e quanto à reutilização (Ms, 2020; Cdcp, 2020).

Levando em consideração a carência dos equipamentos de proteção individual ocasionada pela COVID-19, a reutilização e utilização prolongada dos equipamentos passou a fazer parte do cenário dos serviços de saúde, conforme evidenciam as pesquisas analisadas. Ressalta-se que têm sido viabilizados meios para amenizar o risco dessa prática com os protocolos estabelecidos pelos órgãos de vigilância sanitária, buscando assim barreiras físicas adequadas proporcionadas pelos equipamentos de proteção individual e para a garantia mínima de condições de segurança para o exercício profissional.

O Ministério da Saúde e Anvisa, por exemplo, publicaram uma série de orientações referentes a medidas de saúde e de segurança no trabalho de profissionais da atenção primária à saúde, ligados(as) à média e alta complexidade, considerando equipamentos de proteção individual e coletiva, medidas administrativas, de engenharia dos serviços de saúde e de organização do trabalho (Anvisa, 2020).

O gerenciamento de EPI's, então, deve ser coordenado através de mecanismos básicos de gestão de cadeia de fornecimento, em nível nacional e internacional, que incluam os seguintes itens, sem se limitar, segundo as pesquisas analisadas, a eles: previsões de uso de EPI's que sejam baseadas em modelos racionais de quantificação, de modo a garantir o uso coerente dos itens solicitados; o monitoramento e o controle das solicitações dos equipamentos feitas por países ou grandes centros de resposta ao surto; estímulo ao uso de uma abordagem centralizada de gerenciamento de pedidos para evitar duplicação de 
estoques, para garantir o cumprimento rígido de regras básicas de gerenciamento de estoques como forma de limitar perdas, estoque excessivo ou ruptura de estoque; monitoramento da distribuição dos EPI's de ponta a ponta; além do monitoramento e controle da distribuição de tais equipamentos vendidos em lojas de produtos médicos (Who, 2020).

As recomendações para uso racional de EPI's, no contexto da COVID-19, visam, conforme os estudos mapeados, orientar as pessoas envolvidas na distribuição e no gerenciamento desses equipamentos, bem como auxiliar autoridades de saúde pública e indivíduos que atuam na comunidade e em locais de assistência à saúde, oferecendo guias sobre as situações em que o uso de EPI's é mais adequado. É preciso que haja, então, controle administrativo, ambiental e de engenharia, numa perspectiva de ações e estratégias conjuntas para a efetividade contra a pandemia (Who, 2020).

A evolução da pandemia e, concomitantemente, o potencial aumento de riscos aos(às) trabalhadores(as), impôs ao sistema de saúde a introdução de novas medidas para enfrentamento da problemática envolvendo a escassez de EPI's, buscando assim a organização da logística, o uso racional do equipamento e a proteção eficaz dos(as) trabalhadores(as), almejando a melhor disponibilidade de recursos e a redução na disseminação do contágio pelo COVID-19 entre o referido grupo.

Os(as) trabalhadores(as) podem e devem denunciar a falta de EPI's e rechaçar qualquer pedido de ocultação relacionado à escassez de EPI, pois, infelizmente, há relatos de profissionais de saúde que foram expressamente informados pelas gerências para não falarem publicamente sobre as condições inadequadas em que estão operando, como destacam algumas das pesquisas analisadas. Assim, por medo de retaliações de empregadores, muitos(as) trabalhadores(as) expõem-se diante deste quadro de negligências. Esta grave situação pode indicar, segundo os estudos, que as informações disponíveis sobre a deficiência de EPI's nos serviços de saúde, provavelmente, subestimam o tamanho e extensão do problema (Mandola, 2020; Malhotra, 2020).

O cenário apresentado após a disseminação da COVID-19, conforme a análise feita, é assustador para gestores(as), trabalhadores(as) e comunidade, pois esta situação limite, no que se refere à escassez e qualidade dos EPI's, requer maior empenho, responsabilidade, planejamento e transparências nas tomadas de decisão, para que as vulnerabilidades existentes sejam sanadas, de modo a oferecer melhores condições de trabalho para os(as) profissionais que atuam na linha de frente contra o COVID-19. Deste modo, prestar assistência à população que necessita de atendimento seja de baixa, média ou alta complexidade, é assumida como aspecto central nas pesquisas observadas.

\subsection{O impacto das condições de trabalho e treinamento para utilização do equipamento de proteção individual (EPI) na saúde dos profissionais durante a pandemia}

A pandemia, no Brasil, desvela situações históricas de negligência de políticas públicas, incluindo o subfinanciamento do sistema público de saúde, da ciência, da tecnologia e das universidades públicas, além da desvalorização do trabalho e dos trabalhadores (Cueto, 2020), conforme observado a partir das pesquisas mapeadas.

Os(as) profissionais de saúde convivem, cotidianamente, com condições de trabalho precárias, decorrentes de escassez de recursos e materiais ou de características da organização do trabalho em saúde que envolvem carga de trabalho elevadas, prolongamento de jornadas laborais, trabalho em turnos e dificuldade para pausas e repouso. Embora essas dificuldades se exacerbem em situação de crise, são frequentemente identificadas no trabalho em saúde. Estudos mapeados verificaram que as condições de trabalho ambiental e organizacional afetam a capacidade para o trabalho entre profissionais de saúde (Cordeiro, Araújo, 2016; Godinho, et al., 2017).

Desta forma, a literatura evidencia o descaso que já ocorre há décadas em relação aos(às) profissionais da saúde, no que tange às condições de trabalho, impactando diretamente no desempenho, tomada de decisão e qualidade dos serviços prestados, frente às demandas cotidianas. $\mathrm{O}$ estado de calamidade que os profissionais permeiam acerca das condições de trabalho, foi acentuada pela pandemia da COVID-19, com a intensificação das demandas e exigências de adaptação aos novos protocolos assistenciais. 
Durante surtos e pandemias é comum que profissionais de saúde trabalhem muitas horas, sem pausas e sob grande pressão, levando-os(as) à fadiga e exaustão (Schwartz, Yen, 2020). Geralmente, as condições de trabalho dos(as) profissionais de enfermagem incluem, igualmente, extensas jornadas, ritmo intenso, desvalorização profissional, conflitos interpessoais, entre outros fatores desencadeantes de desgastes físicos e psíquicos (Santana, 2018).

Ademais, destaca-se o contexto das emergências, que diante da pandemia também são marcados pela falta de recursos materiais, escassez de força de trabalho, excesso de atividades laborais e elevada demanda de pacientes, além de condições físicas inadequadas para o processo de trabalho. Essas situações, segundo a literatura em questão, afetam diretamente a autoestima do(a) profissional e sua estrutura emocional, contribuindo para que a equipe de enfermagem esteja exposta à diminuição de estímulo para exercer suas funções e que aflorem sentimentos de impotência (Santos, et al., 2019).

Desse modo, dispor da capacidade plena para desenvolver as atribuições são requisitos para a prática assistencial eficiente. Os(as) profissionais necessitam de repouso com tempo e local adequado para regenerar-se do desgaste físico e psíquico, sobretudo para a adequada duração da jornada de trabalho, para minimizar a exposição ao agente infeccioso, especialmente em uma pandemia, que demanda maior atenção e agilidade na tomada de decisão. Levando em consideração a segurança dos(as) trabalhadores(as) e pacientes, o desgaste físico e psíquico eleva o risco para erros na prestação da assistência, podendo estes ocasionar graves complicações a trabalhadores(as) e pacientes. Além disso, remuneração, condições físicas e falta de materiais, potencializam para o esgotamento e desmotivação desses(as) trabalhadores(as), refletindo diretamente na qualidade da assistência prestada, conforme mostram os estudos analisados.

Contudo, a situação atual de crise sanitária evidencia o papel crucial desses(as) trabalhadores(as) para a garantia da vida das populações afetadas. Pandemias exigem prontamente que serviços de saúde respondam às demandas às quais nem sempre estão preparados. Durante uma epidemia, a organização do trabalho de diversos grupos ocupacionais sofre profundas alterações quanto à jornada de trabalho, realização de horas extras e ritmo de trabalho (Schwartz, Yen, 2020).

Neste contexto, é imprescindível rever os danos que as condições de trabalho causam aos(às) trabalhadores(as) da saúde, levando em consideração o serviço por eles(as) prestado, que os(as) tornam protagonistas em meio ao quadro causado pela COVID-19, e fez o mundo enxergar a necessidade do trabalho eficaz mesmo com condições adversas, mas que salvou diversas vidas onde o panorama era devastador.

Neste cenário, a importância da vida dos(as) profissionais de enfermagem e sua responsabilidade civil entram em conflito, podendo trazer consequências para sua saúde e segurança no desempenho de suas atividades laborais. Faz-se necessário e urgente que os(as) profissionais de enfermagem tenham EPI's adequados e sejam capacitados em serviços para o seu uso correto (Huang, et al.; 2020).

Desta forma, outro fator que desencadeia apreensão aos(às) profissionais, segundo a literatura, trata-se da capacitação/treinamento para utilização dos EPI's, frente a pacientes com suspeita ou confirmação de contaminação por COVID19, para diminuir o risco de contaminação e disseminação entre os(as) profissionais de saúde no desenvolvimento das atividades laborais cotidianas.

As orientações sobre o uso de EPI's podem ter diversas interpretações e divergir na indicação, como em relação ao uso de macacão ou capote impermeável para a assistência ao(à) paciente infectado(a). Estas ações se justificam, conforme estudos mapeados, pela diversidade de informações e pelo distanciamento de protocolos, guidelines e experiências internacionais (Adams, Walls, 2020). O repasse de orientações sobre as medidas de prevenção é essencial para gerenciamento do estresse causado pelas atividades laborais (Huang, et al.; 2020).

No entanto, o treinamento convencional utilizando demonstrações não garante que o(a) profissional se paramente e desparamente de forma adequada. Um estudo acerca de treinamento e educação, no contexto de doenças altamente infecciosas, em serviços de emergência, demonstra, por exemplo, que profissionais sentiam a necessidade de treinamentos de qualidade e 
falta de confiança suficiente para responderem a estas doenças (Le, et al.; 2020).

As orientações para a paramentação e desparamentação na pandemia de COVID-19, trouxe à tona diversos embates entre os(as) trabalhadores(as), devido às diversas informações em desalinho, o que dificulta no entendimento e padronização para realização da técnica adequada conforme recomendação científica. Outra questão, destaca a necessidade de os serviços de saúde ofertarem treinamento com efetiva prática da técnica, buscando a elucidação de dúvidas e aperfeiçoamento das habilidades necessárias para paramentação e desparamentação adequada.

Portanto, o investimento no conhecimento, a capacitação e treinamento dos(as) profissionais da área da saúde sobre o uso adequado desses equipamentos no manejo e cuidado dos(as) pacientes infectados(as) torna-se imprescindível. A paramentação e desparamentação de maneira adequada é uma forma eficaz de se evitar contaminação entre os(as) profissionais da área da saúde (Anvisa, 2020).

Levantamentos preliminares realizados por conselhos profissionais e investigações científicas apresentam um cenário preocupante. Pesquisa conduzida em hospitais públicos da cidade do Rio de Janeiro, nesse sentido, identificou elevada taxa de infecção pelo novo coronavírus entre profissionais de saúde (25\%), muito superior àquelas verificadas na China (4\%) e Itália (15\%) em estágios mais avançados da pandemia (Azevedo, 2020; Hok, 2020; Anelli, et al.; 2020).

Em maio, o Conselho Federal de Enfermagem e o Conselho Internacional de Enfermagem (ICN) afirmaram, a partir de importante estudo, que o Brasil é o país com mais mortes de enfermeiros(as) e profissionais de saúde devido à pandemia de COVID-19. De acordo com o COFEN, foram, até aquele momento, 157 mortes de profissionais de enfermagem. O INC informa que o país tem um número de mortes entre enfermeiros(as) maior que o dos Estados Unidos, com 146 óbitos, e que o do Reino Unido, com 77 (Cofen, 2020).

O fato é que, em se tratando de doença com elevado potencial de transmissibilidade e aumento desordenado no número de pacientes que buscam atendimento nos serviços de saúde, investimento em treinamento é indispensável para manter contingente de profissionais necessário para atuação e preservação da vida e saúde desses(as) trabalhadores(as), resultando na melhor vigilância e segurança destes(as) no ambiente de trabalho.

A literatura reforça, então, que aumento da conscientização sobre proteção pessoal, fornecimento de EPI's adequados, em número suficiente, com treinamento de acordo com protocolos nacionais e internacionais, pode contribuir para redução do risco de infecção em profissionais de saúde (Wang, Zhou, Liu, 2020).

A melhoria nas informações/treinamento, considerandoas evidências científicas disponíveis, faz-se necessário, segundo os estudos, para enfrentamento da epidemia e subdimensionamento deste problema que ceifa ou causa danos, muitas vezes irreparáveis, aos(às) trabalhadores(as) que atuam na linha de frente contra COVID-19 e que necessitam de ações efetivas e imediatas para melhoria deste cenário. Segundo a literatura em questão, é necessário que estes se sintam amparados, protegidos e reconhecidos pela relevância do serviço prestado à população, numa luta muitas vezes "desleal" e "interminável" contra a COVID-19.

\section{Conclusão}

Este estudo pleiteou enfatizar o detrimento ao sistema de saúde e trabalhadores/as que se encontram na linha de frente contra o covid-19, frente à escassez e qualidade do equipamento de proteção individual, condições de trabalho e treinamento ao longo da pandemia.

Há inúmeros motivos para se afirmar que os trabalhadores/as da saúde permanecem mais vulneráveis frente a pandemia, levando em consideração a fragilidade e incapacidade do sistema de gestão, que há anos é objeto de manobras inadequadas e de 
gestores incapacitados e mal intencionados. A falta de planejamento logístico para compra, distribuição e armazenamento de recursos materiais, com a quantidade e qualidade adequadas, vem colocando em risco a vida de trabalhadores/as em todo o sistema de saúde que atuam no combate a pandemia de COVID-19. Mais ainda, a escassez do material necessário para prestação da assistência a pacientes com suspeita ou confirmação de COVID-19, mostra o abismo que os trabalhadores/as da saúde se encontram mediante a gravidade e complexidade da situação que se instalou desordenadamente nas diferentes áreas da assistência.

A falta de organização, despreparo para planejar e ajustar ações imediatas para suprir a demanda dos equipamentos de proteção individual, denunciou o colapso do sistema de saúde frente a pandemia de covid-19 e o descaso com a segurança dos trabalhadores/as que se tornaram vítimas dos desmandos destes gestores, assim como toda a população que necessita deste serviço. A atuação dos/as trabalhadores/as da saúde tem papel fundamental na realização deste trabalho, necessitando de condições adequadas para proteção, segurança e manutenção da sua qualidade de vida. Estes direitos básicos para execução do trabalho tornam-se como objetivo a ser alcançado nas ações de enfrentamento da epidemia. O cenário apresentado, destaca problemas, inúmeras vezes apontados na literatura, com evidências sólidas para tomadas de decisões mais contundentes e eficazes, buscando melhores condições de trabalho, remuneração, jornada de trabalho e segurança para desenvolver suas atividades laborais.

\section{Referências}

Adams, J. G., \& Walls, R. M. (2020). Supporting the Health Care Workforce During the COVID-19 Global Epidemic. JAMA. 32, 3. http://doi.org/doi:10.1001/jama.2020.3972.

Agência Nacional de Vigilância Sanitária. (2020). Nota Técnica GVIMS/GGTES/ANVISA nº5/2020: Orientações para serviços de saúde: Medidas de prevenção e controle que devem ser adotadas durante a assistência aos casos suspeitos ou confirmados de infecção pelo novo coronavírus (SARSCoV-2). Brasília, ANVISA. https://www20.anvisa.gov.br/segurancadopaciente/index.php/alertas/category/covid-19.

Agência Nacional de Vigilância Sanitária. Nota Técnica 04/2020 Anvisa. (2020). http://portal.anvisa.gov.br/servicos/notas-tecnicas.

Agência Nacional de Vigilância Sanitária. Nota técnica GVIMS/GGTES/ANVISA nº 04/2020. (2020). Orientações para serviços de saúde: medidas de prevenção e controle que devem ser adotadas durante a assistência aos casos suspeitos ou confirmados de infecção pelo novo coronavírus (SARS-COV-2). http://portal.anvisa.gov.br/documents/33852/271858/Nota+T\%C3\%A9cnica+n+04-2020+GVIMS-GGTES-ANVISA/ab598660-3de4-4f14-8e6fb9341c196b28.

Anelli, F., et al (2020). Italian doctors call for protecting healthcare workers and boosting community surveillance during covid-19 outbreak. BMJ, 368 : m1254.

Associação Brasileira de Medicina. (2020). Faltam EPIs em todo país. http://amb.org.br/epi/.

Azevedo, A. L. (2020). Coronavírus atinge até $25 \%$ de profissionais de saúde no Rio. O Globo, Seção Sociedade, Rio de Janeiro, 8 abr. http://oglobo.globo.com/sociedade/coronavurus/coronavirus-atinge-ate-25-dos-profissionais-de-saude-do-rio-1-24357939.

Centers for Disease Control and Prevention. (2020). Factors to Consider When Planning to Purchase Respirators from Another Country. https://www.cdc.gov/coronavirus/2019-ncov/hcp/ppe-strategy/internationalrespirator-purchase.html.

Centers for Disease Control and Prevention. (2020). Interim Infection Prevention and Control Recommendations for Patients with Suspected or Confirmed Coronavirus Disease 2019 (COVID-19) in Healthcare Settings. https://www.cdc.gov/coronavirus/2019-ncov/hcp/infection-control-recommendations.html.

Centers for Disease Control and Prevention. (2020). Strategies for Optimizing the Supply of N95 Respirators. https://www.cdc.gov/coronavirus/2019ncov/hcp/respirators-strategy/index.html.

Chang, D., et al. (2020). Protecting health-care workers from subclinical coronavirus infection. Lancet. Published Online February 13. https://doi.org/10.1016/S2213-2600(20)30066-7.

Conselho Federal de Enfermagem. (2020). Brasil é o país com mais mortes de enfermeiros por COVID-19 no mundo. http://www.cofen.gov.br/brasil-e-0-paiscom-mais-mortes-de-enfermeiros-por-covid-19-no-mundo-dizem-entidades_80181.html.

Cordeiro, T. M. S., \& Araújo, T. (2016). Capacidade para o trabalho e fatores associados em profissionais de saúde no Brasil. Revista Brasileira Medicina do Trabalho, São Paulo, 14(3), 262-74.

Cueto, M. (2020). O COVID-19 e as epidemias da globalização. História, Ciências e Saúde- Manguinhos, Capa, 29 mar 2020. http://www.revistahcsm.coc.fiocruz.br/o-covid-19-e-as-epidemias-da-globalizacao/.

Ferioli, M., et al. (2020). Protecting healthcare workers from SARS-CoV-2 infection: practical indications. European Respiratory Review. https://doi.org/10.1183/16000617.0068-2020. 
Fundação Oswaldo Cruz. (2020). Fiocruz produzirá kits para diagnóstico do novo coronavírus. Rio de Janeiro, Fundação Oswaldo Cruz. https://portal.fiocruz.br/noticia/ fiocruz-produzira-kits-para-diagnostico-do-novo-coronavirus.

Gallasch, C. H., et al. (2020). Prevention related to the occupational exposure of health professionals workers in the COVID-19 scenario. Rev. enferm. UERJ, 28: e49596. https://www.epublicacoes.uerj.br/index.php/enfer magemuerj/article/view/49596/33146.

Godinho, M. R. et al. (2017). Capacidade para o trabalho e fatores associados em profissionais no Brasil. Revista Brasileira de Medicina do Trabalho, São Paulo, 15(1), 88-100.

Guo, Y. R., et al. (2020). The origin, transmission and clinical therapies on coronavirus disease 2019 (Covid-19) outbreak: an update on the status. Mil Med Res. 2020, 7(1):1-10. https://doi.org/10.1186/s40779-020-00240-0.

Holland, M., Zaloga, D. J., \& Friderici, C. S. (2020). COVID-19: Personal Protective Equipment (PPE) for the emergency physician. Vis J Emerg Med., 19:100740. 10.1016/j.visj.2020.100740.

Huang, L., et al. (2020). Special attention to nurses' protection during the COVID-19 epidemic. Critical Care, 24 . https://ccforum.biomedcentral.com/articles/10.1186/s13054-020-2841-7.

Janssen, L., Zhuang, Z, \& Shaffer, R. (2014). Criteria for the collection of useful respirator performance data in the workplace. J Occup Environ Hyg., 11(4), 218-226. https://www.ncbi.nlm.nih.gov/pmc/articles/PMC4739800/pdf/nihms753016.pdf.

Jin, Y. H., et al. (2020). A rapid advice guideline for the diagnosis and treatment of 2019 novel coronavirus (2019-nCoV) infected pneumonia (standard version). Military Medical Research, v. 7, (1):1-23. https://doi.org/10.1186/s40779-020-0233-6.

Koh, D. (2020). Occupational risks for COVID-19 infection. Occupational Medicine (Oxford, England), 70(1), 3-5.

Lauer, S. A., et al. (2020). The Incubation Period of Coronavirus Disease 2019 (COVID-19) From Publicly Reported Confirmed Cases: Estimation and Application. Annals of internal medicine, 172, 9, 577-582. 10.7326/M20-0504.

Le, A., et al. (2018). Determining training and education needs pertaining to highly infectious disease preparedness and response: a gap analysis survey of US emergency medical services practitioners. Am J Infect Control. 3, 246-52. 10.1016/j.ajic.2017.09.024

Malhotra, N. (2020). COVID-19 in intensive care. Some necessary steps for health care workers. Monaldi Arch Chest Dis. 2020;25(1):90. 10.4081/monaldi.2020.1284.

Mandola, J. (2020). CoViD-19 e dispositivi di protezione individuale: qualcuno di noi morirà per la loro carenza. Recenti Prog Med, 111:183. https://www.recentiprogressi.it/r.php?v=3347\&a=33175\&l=340294\&f=allegati/03347_2020_04/fulltext/04_Editoriale\%20-\%20Mandrola.pdf.

Ministério da Saúde. (2006). Biossegurança em Laboratórios Biomédicos e de Micro-biologia. Brasília, Ministério da Saúde.

Ministério da Saúde. (2009). Portaria No 2.728 de 11 de novembro de 2009: Dispõe sobre a Rede Nacional de Atenção Integral à Saúde do Trabalhador. Brasília, MS. http://bvsms.saude.gov.br/bvs/saudelegis/gm/2009/prt2728_11_11_2009.html

Ministério da Saúde. (2012). Portaria No 1.823 de 23 de agosto de 2012: institui a Política Nacional de Saúde do Trabalhador e da Trabalhadora. Brasília, MS.

Ministério da Saúde. (2020). Recomendações de proteção aos trabalhadores dos serviços de saúde no atendimento de COVID-19 e outras síndromes gripais. https://portalarquivos.saude.gov.br/images/pdf/2020/April/16/01-recomendacoes-de-protecao.pdf.

Ministério da Saúde. (2020). Recomendações de proteção aos trabalhadores dos serviços de saúde no atendimento de COVID-19 e outras síndromes gripais. [Internet]. 2020. https://portalarquivos.saude.gov.br/images/pdf/2020/April/16/01-recomendacoes-de-protecao.pdf.

Ministério da Saúde. (2020). Resolução - RDC no 356, de 23 de março de 2020. http://www.in.gov.br/en/web/dou/-/resolucao-rdc-n-356-de-23-de-marco-de2020-249317437.

Ministério da Saúde. Agência Nacional de Vigilância Sanitária. (2009). Segurança do Paciente em Serviços de Saúde: higienização das mãos. Brasília, Anvisa.

Ministério da Saúde. Agência Nacional de Vigilância Sanitária. (2020). Nota Técnica GVIMS/GGTES/ANVISA $n^{\circ}$ 05/2020: Orientações para serviços de saúde: Medidas de prevenção e controle que devem ser adotadas durante a assistência aos casos suspeitos ou confirmados de infecção pelo novo Coronavírus (SARSCoV-2) em instituições de longa permanência para idosos. Brasília, ANVISA.

Ministério da Saúde. Agência Nacional de Vigilância Sanitária. (2020). Nota Técnica GVIMS/GGTES/ANVISA Nº4/2020. Orientações para serviços de saúde: medidas de prevenção e controle que devem ser adotadas durante a assistência aos casos suspeitos ou confirmados de infecção pelo novo coronavírus (SARSCov-2). http://portal.anvisa.gov.br/documents/33852/271858/Nota+T\%C3\%A9cnica+n+04-2020+GVIMS-GGTES-ANVISA/ab598660-3de4-4f14-8e6fb9341c196b2.

Ministério da Saúde. Secretaria de Vigilância em Saúde. (2020). Guia de vigilância epidemiológica para infecção humana para o COVID-19. Brasília, Ministério da Saúde. https://portalarquivos.saude.gov.br/images/pdf/2020/April/07/GuiaDeVigiE-pidemC19-v2.pdf.

Ministério da Saúde. Secretaria de Vigilância em Saúde. (2020). Recomendações de proteção aos trabalhadores dos serviços de saúde no atendimento da COVID-19 e outras síndromes gripais. Brasília, Ministério da Saúde. https://portalarquivos.saude.gov.br/images/pdf/2020/April/16/01-recomendacoesdeprotecao.pdf.

Ministério do Trabalho e Emprego. (2005). Norma Regulamentador n. 32, de 11 de novembro de 2005. Segurança e Saúde no Trabalho em Serviços de Saúde. Brasília, MS. 
Research, Society and Development, v. 10, n. 2, e55510212772, 2021

(CC BY 4.0) | ISSN 2525-3409 | DOI: http://dx.doi.org/10.33448/rsd-v10i2.12772

Ministério do Trabalho e Emprego. (2013). Norma Regulamentadora N. 6. Equipamento de Proteção Individual. EPI. www.portal.mte.gov.br/data/files/.../NR06\%20(atualizada)\%202010.pdf.

Ministério do Trabalho e Emprego. (2014). Portaria No 590 de 28 de abril de 2014: Norma Regulamentadora 4 - Serviços Especializados em Engenharia de Segurança e em Medicina do Trabalho. Brasília, MTE.

Ministério do Trabalho e Emprego. (2020). Norma regulamentadora $n^{\circ} 32$ : segurança e trabalho nos estabelecimentos de saúde. Portaria de $N^{\circ} 485$, de 11 de novembro de 2005. http://www.anvisa.gov.br/servicosaude/avalia/saude_do_trabalhador_portaria_485_aprovaNR32.pdf.

Ministério do Trabalho. (2013). Norma Regulamentadora NR-7. Programa de controle médico de saúde ocupacional. https://enit.trabalho.gov.br/portal/ima ges/ Arquivos_SST/SST_NR/NR-07.pdf.

Santana, L. de L. (2018). Riscos psicossociais e saúde mental em ambiente hospitalar: com a voz o trabalhador [tese]. Curitiba (PR), Universidade Federal do Paraná.

Santos, J. N. M. O. (2019). Estresse ocupacional: exposição da equipe de enfermagem de uma unidade de emergência. J. Res. Fundam. Care. 2019; 11:455-63. http://www.dx.doi.org/10.9789/2175-531.2019.v11i2.455-463.

Schwartz, J. K., Chwan-Chuen, Y. Muh-Yong. (2020) Protecting Healthcare Workers During the Coronavirus Disease (COVID19) Outbreak: Lessons from Taiwan's Severe Acute Respiratory Syndrome Response. Clinical Infectious Diseases. ciaa255, 1-3, 2020.

Soares, C. B. (2014). Integrative review: Concepts and methods used in Nursing. Revista da Escola de Enfermagem USP, 48(2), 335-345, 2014. https://doi:10.1 590/S0080-6234201400002000020.

Souza, M. T. De., Silva, M, D. Da., \& Carvalho, R. de. (2010). Revisão integrativa: o que é e como fazer. Einstein (São Paulo), 8(1), 102-106, mar. http://www.scielo.br/scielo.php?script=sci_arttext\&pid=S1679-45082010000100102\&lng=en\&nrm=iso.

Wang, J., Zhou, M., \& Liu, F. (2020). Reasons for healthcare workers becoming infected with novel coronavirus disease 2019 (COVID-19) in China. J Hosp Infect. https://doi.org/10.1016/j.jhin.2020.03.002.

World Health Organization. (2020). Cuidados para profissionais da saúde expostos ao novo coronavírus (COVID-19) em estabelecimentos de saúde. https://iris.paho.org/bitstream/handle/10665.2/52050/OPASBRACOVID1920046_por.pdf?sequence=1\&isAllowed=y

World Health Organization. (2020). Rational use of personal protective equipment (PPE) for coronavirus disease (COVID-19). https://apps.who.int/iri s/bitstream/ handle/10665/331498/WHO-2019-nCoV-IPCPPE_use-2020.2-eng.pdf.

World Health Organization. (2020). Requirements and technical specifications of personal protective equipment (PPE) for the novel coronavirus (2019-ncov) in healthcare settings. https://iris.paho.org/bitstream/handle/10665.2/51906/requirements-\%20PPE-coronavirus-eng.pdf?sequence=1\&isAllowed=y.

World Health Organization. (2020). WHO Director-General's opening remarks at the media briefing on COVID-19. https://www.who.int/dg/speech es/detail/who-director-general-s-opening-remarks-at-the-media-briefing-on-COVID-19.

Zhang, J., et al. (2020). Therapeutic and triage strategies for 2019 novel coronavirus disease in fever clinics. Lancet Respir Med. 8, (3):11-12. https://doi.org/ $10.1016 / \mathrm{S} 2213-2600(20) 30071-0$ 